\title{
„Wer sich qualifiziert und an der Zertifizierung teilnimmt, verbessert seine Berufschancen“
}

\author{
Das DeGIR-/ DGNR-Zertifizierungsprogramm ist nicht nur für \\ langjährig tätige Interventionalisten gedacht, schon in der Wei- \\ terbildungszeit kann gezielt am Erwerb der Zertifikate gearbei- \\ tet und die Karriere geplant werden, sagt PD Dr. Peter Land- \\ wehr (Hannover), einer der Begründer der Initiative für Qualifi- \\ zierung und Zertifizierung in der Interventionellen Radiologie \\ und Neuroradiologie.
}

Herr Dr. Landwehr, wie zufrieden sind Sie mit der Aufnahme des Programms unter den Mitgliedern?

Landwehr: Wir sind überwältigt von der Resonanz. Sowohl die Fortbildungsangebote als auch die Zertifizierung selbst werden sehr gut angenommen. Seit Start des Programms 2010 haben wir knapp 900 Zertifizierungen erteilen können. Das zeigt uns, dass wir den Nerv getroffen haben. Mir ist es ein besonderes Anliegen, das Programm gerade auch unter den Nachwuchsradiologen bekannter zu machen. Die Teilnahme am Programm ist auch unterhalb des Expertenlevels möglich als Stufe 1-Zertifikat, und die Voraussetzungen können schon während der Weiterbildungszeit erworben werden. Auch für die thematisch definierten Module der Stufe 2 können vor der Facharztprüfung erste Voraussetzungen wie Eingriffszahlen oder CME-Punkte gesammelt werden.

Welchen konkreten Nutzen, welche - auch karrieretechnischen - Vorteile bringt die Teilnahme?

Landwehr: Alle profitieren von dem Programm. Der Arbeitgeber profitiert von der strukturierten Qualifizierung der Mitarbeiter, und er bekommt eine sehr gute Handhabe für das Recruiting. Wir sehen, dass in den Stellenausschreibungen immer häufiger die DeGIR/ DGNR-Zertifizierung in das Anforderungsprofil aufge- nommen wird. Dann profitieren die radiologischen Abteilungen von dem Programm: Es stärkt die Radiologie im internen Wettbewerb mit anderen Gefäßdisziplinen oder hilft bei der Diskussion um größere Investitionen, etwa wenn es um die Anschaffung eines Hybrid-OPs geht. Und schließlich profitiert der interventionell tätige Radiologe von einer transparenten und strukturierten Fortbildung, den vielfältigen Möglichkeiten der Höherqualifizierung durch Spezialkurse und die genannten Profilierungen gegenüber anderen Kollegen und Abteilungen. Wer frühzeitig die Qualifizierungsangebote nutzt und dann an der Zertifizierung teilnimmt, verbessert sein interventionelles Können und seine Berufschancen.

Welche Bedeutung hat das Zertifizierungsprogramm - in wissenschaftlicher und versorgungspolitischer Hinsicht?

Landwehr: Die Interventionelle Radiologie hat in den vergangenen Jahren einen enormen Aufschwung erfahren. Sie ist heute DIE führende Disziplin in der durch Bildgebung gesteuerten minimalinvasiven Therapie. Die Aufgaben, die sich aus dieser mittlerweile flächendeckenden Versorgung ergeben, sind enorm. Wir müssen junge Radiologinnen und Radiologen an die Verfahren heranführen, wir müssen Hospitationsmöglichkeiten schaffen, denn nicht jeder Eingriff kann an jedem Haus gelernt werden. Das Zertifi-

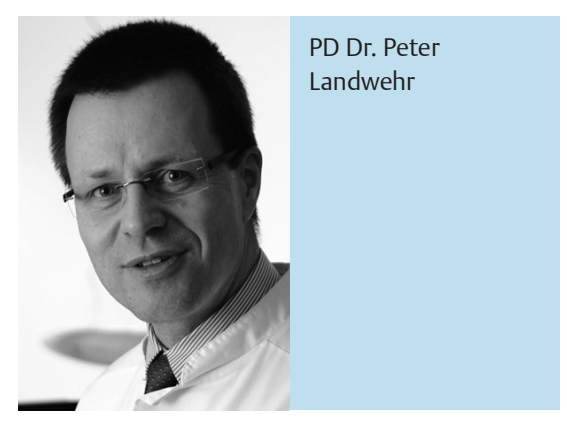

zierungsprogramm leistet hier einen wichtigen Beitrag, die Versorgungsqualität zu heben und zu halten. Es führt langfristig zu einer Verbesserung der Versorgungsstrukturen - etwa bei der Therapie der pAVK, des Schlaganfalls und in der interventionellen Onkologie - und es weckt das Interesse des radiologischen Nachwuchses, was sich wiederum günstig auf wissenschaftliche Aktivitäten auswirken wird, gerade im Umfeld von Universitätskliniken.

\section{Welche Weiterentwicklungen sind geplant?}

Landwehr: Noch stärker als bislang wollen wir die kurrikulären Kursangebote, die Basis- und die Spezialkurse für die Module, auf den etablierten Kongressen wie dem Deutschen Röntgenkongress und dem IROS anbieten und die Angebote besser verzahnen. Wir planen das Kursprogramm um weitere Medien, DeGIR-geprüfte Lehrbücher und Lehrfilme auszuweiten. Und wir werden das Hospitationsnetz ausbauen und bewerben.

\section{Weiterführende Informationen un-}

ter:

http://www.degir.de/site/degir-dgnr-modul-und-stufenkonzept

http://www.drgakademie.de/deDE/1103/degir-akademie

http://www.hellste-koepfe.de/site/facharzt/facharzt_und_pruefung/zertifizierung-interventionen

\section{Pressekontakt:}

Florian Schneider

Deutsche Röntgengesellschaft e.V.

Tel.:030-916070 19

Fax: 030-916 07022

E-Mail: schneider@drg.de |

Internet: $w w w . d r g . d e$ 